$\underline{\xi}=\mathbf{Z}$

\title{
On the stability of ${ }_{L_{4,5}}$ in the perturbed relativistic $\mathbf{R 3 B P}$ with a triaxial bigger primary
}

\author{
Nakone Bello $^{1}$, Jagadish Singh ${ }^{2}$ \\ ${ }^{1}$ Department of Mathematics, Faculty of Science Usmanu Danfodiyo University, Sokoto, Nigeria \\ ${ }^{2}$ Department of Mathematics, Faculty of Science, Ahmadu Bello University, Zaria. Nigeria \\ *Corresponding author E-mail: bnakone@yahoo.com
}

\begin{abstract}
In the present paper, we endeavor to study the stability of triangular points under the influence of small perturbations in the Coriolis and centrifugal forces, together with the triaxiality of the bigger primary in the framework of the relativistic R3BP. It is observed that the locations of these points are affected by the relativistic factor, triaxiality and a small perturbation in the centrifugal force, but are unaffected by that of the Coriolis force. It is also seen that for these points the range of stability region increases or decreases according as equation (14) without $\mu_{0}$ is greater or less than zero.
\end{abstract}

Keywords: Celestial Mechanics; Perturbation; Relativity; Triaxiality; R3BP.

\section{Introduction}

The restricted three-body problem (R3BP) in which two massive bodies (primaries) revolve around their common center of mass in circular orbits and a third body of negligible mass moves in their gravitational field, is a simple problem and has been receiving considerable attention of scientists and astronomers because of its applications in dynamics of the solar and stellar systems, lunar theory and artificial satellites. It possesses five points of equilibrium: three collinear and two triangular, where the gravitational and centrifugal forces just balance each other. The collinear libration points $\mathrm{L}_{1}, \mathrm{~L}_{2}, \mathrm{~L}_{3}$ are unstable, while the two equilateral libration points $\mathrm{L}_{4}, \mathrm{~L}_{5}$ are stable for $\mu<\mu_{\text {crit }}=0.038520 \ldots$ Szebehely [1] The stability occurs in spite of the fact that the potential energy has a maximum rather than a minimum at the latter points. The stability is actually achieved through the influence of the Coriolis force, because the coordinate system is rotating (Wintner [2]; Contopolous [3]).

By taking small perturbations in the Coriolis and centrifugal forces, various authors (Szebehely [4]; Bhatnagar and Hallan [5]; AbdulRaheem and Singh [6]; Singh and Begha [7]; Abouelmagd et al. [8]; Singh [9]) have described their effects on the motion of the third body. Szebehely [4] investigated the stability of triangular points by keeping the centrifugal force constant and found that the Coriolis force is a stabilizing force. This was confirmed by AbdulRaheem and Singh [6].

The bodies in the R3BP are strictly spherical in shape, but in nature, celestial bodies are not perfect spheres. They are either oblate or triaxial. The Earth, Jupiter, Saturn, Regulus, Neutron stars and black dwarfs are oblate. The Moon, Pluto and its moon Charon are triaxial. The lack of sphericity, triaxiality or oblateness of the celestial bodies causes large perturbations in a two -body orbit. Brumberg [10], [11] studied the relativistic $\mathrm{n}$-body problem of three bodies in more details and collected most of the important results on relativistic celestial mechanics. He did not only obtain the equations of motion for the general problem of three bodies but also deduced the equations of motion for the restricted problem of three bodies.

Bhatnagar and Hallan [12] studied the existence and linear stability of the triangular points $\mathrm{L}_{4,5}$ in the relativistic $\mathrm{R} 3 \mathrm{BP}$, and found that $L_{4,5}$ are always unstable in the whole range $0 \leq \mu \leq \frac{1}{2}$ in contrast to the classical R3BP where they are stable for $\mu<\mu_{0}$, where $\mu$ is the mass ratio and $\mu_{0}=0.03852 \ldots$ is the Routh's value.

Douskos and Perdios [13] investigated the stability of the triangular points in the relativistic R3BP and contrary to the results of Bhatnagar and Hallan [12], they obtained a region of linear stability in the parameter space $0 \leq \mu<\mu_{0}-\frac{17 \sqrt{69}}{486 \mathrm{c}^{2}}$ where $\mu_{0}=0.03852 \ldots$

is Routh's value. They also determined the positions of the collinear points and showed that they are always unstable. In recent times, many perturbing forces i.e. oblateness, radiation of the primaries, centrifugal and Coriolis forces have been included in the study of the relativistic R3BP.

Katour et al. [14] studied the locations of the triangular points within the frame work of the post-Newtonian approximation when the mass of the primaries are assumed to change under the effect of continuous radiation process and oblateness effects of the two primaries. New perturbed locations of the triangular points are computed.

Singh and Bello [15] examined the effect of centrifugal force in the relativistic R3BP and noticed that the positions and stability of the triangular points are affected by both the relativistic factor and a small perturbation in the centrifugal force. Singh and Bello [16] studied the stability of the relativistic R3BP with small perturbations in the centrifugal and Coriolis without including coupling terms $\frac{\varepsilon_{i}}{c^{2}}(i=1,2), \varepsilon_{1}, \varepsilon_{1}$ are the small perturbations in the centrifu- 
gal and Coriolis forces respectively and $\mathrm{c}$ is the speed of light. Our aim is to study the combined effect relativistic factors, triaxiality of the bigger primary and small perturbations in the Coriolis and centrifugal forces on the positions and linear stability of the triangular points by considering coupling terms $\frac{\varepsilon_{i}}{c^{2}}(i=1,2)$ too.

This paper is organized as follows: In Sect. 2, the equations governing the motion are presented; Sect. 3 describes the positions of triangular points, while their linear stability is analyzed in Sect.4 the discussion is given in Sect. 5, finally sect. 6 summarizes the results of this paper.

\section{Equations of motion}

The pertinent equations of motion of an infinitesimal mass in the relativistic R3BP when the bigger primary is triaxial in a barycentric synodic coordinate $\operatorname{system}(\xi, \eta)$ and dimensionless variables can be written as Brumberg [11] and, Singh and Bello [16]:

$$
\begin{aligned}
& \ddot{\xi}-2 \phi n \dot{\eta}=\frac{\partial \mathrm{W}}{\partial \xi}-\frac{\mathrm{d}}{\mathrm{dt}}\left(\frac{\partial \mathrm{W}}{\partial \dot{\xi}}\right) \\
& \ddot{\eta}+2 \phi n \dot{\xi}=\frac{\partial \mathrm{W}}{\partial \eta}-\frac{\mathrm{d}}{\mathrm{dt}}\left(\frac{\partial \mathrm{W}}{\partial \dot{\eta}}\right)
\end{aligned}
$$

Where,

$$
\begin{aligned}
& \mathrm{W}=\frac{1}{2}\left(1+\frac{3}{2}\left(2 \sigma_{1}-\sigma_{2}\right)\right) \psi\left(\xi^{2}+\eta^{2}\right)+ \\
& \frac{(1-\mu)}{\rho_{1}}+\frac{\mu}{\rho_{2}}+\frac{1-\mu}{2 \rho_{1}^{3}}\left(2 \sigma_{1}-\sigma_{2}\right)++\frac{3(1-\mu) \eta^{2}}{2 \rho_{1}^{5}}\left(\sigma_{2}-\sigma_{1}\right) \\
& +\frac{1}{\mathrm{c}^{2}}\left[-\frac{3}{2}\left\{1-\frac{1}{3} \mu(1-\mu)\right\}\left(\xi^{2}+\eta^{2}\right) \psi+\right. \\
& \frac{1}{8}\left\{\phi\left(\dot{\xi}^{2}+\dot{\eta}^{2}\right)+2 \phi(\xi \dot{\eta}-\eta \dot{\xi})+\psi\left(\xi^{2}+\eta^{2}\right)\right\}^{2} \\
& +\frac{3}{2}\left(\frac{1-\mu}{\rho_{1}}+\frac{\mu}{\rho_{2}}\right)\left\{\begin{array}{l}
\phi\left(\dot{\xi}^{2}+\dot{\eta}^{2}\right)+2 \phi(\xi \dot{\eta}-\eta \dot{\xi})+ \\
\psi\left(\xi^{2}+\eta^{2}\right)
\end{array}\right\}-\frac{1}{2}\left(\frac{(1-\mu)^{2}}{\rho_{1}^{2}}+\frac{\mu^{2}}{\rho_{2}^{2}}\right) \\
& +\mu(1-\mu)\left\{\left(4 \phi \dot{\eta}+\frac{7}{2} \psi \xi\right)\left(\frac{1}{\rho_{1}}-\frac{1}{\rho_{2}}\right) \frac{\eta^{2}}{2} \psi\left(\frac{\mu}{\rho_{1}^{3}}+\frac{1-\mu}{\rho_{2}^{3}}\right)+\right. \\
& \left.\left.\psi\left(\frac{-1}{\rho_{1} \rho_{2}}+\frac{3 \mu-2}{2 \rho_{1}}+\frac{1-3 \mu}{2 \rho_{2}}\right)\right]\right]
\end{aligned}
$$

And $\mathrm{n}$ the perturbed mean motion of the primaries is given by

$$
\begin{aligned}
& \mathrm{n}=1+\frac{3}{4}\left(2 \sigma_{1}-\sigma_{2}\right)-\frac{3}{2 c^{2}}\left(1-\frac{1}{3} \mu(1-\mu)\right) \\
& \rho_{1}^{2}=(\xi+\mu)^{2}+\eta^{2} \\
& \rho_{2}^{2}=(\xi+\mu-1)^{2}+\eta^{2}
\end{aligned}
$$

where $0<\mu \leq \frac{1}{2}$ is the ratio of the mass of the smaller primary to the total mass of the primaries, $\rho_{1}$ and $\rho_{2}$ are distances of the infinitesimal mass from the bigger and smaller primary, respectively; $\mathrm{n}$ is the mean motion of the primaries; $\mathrm{c}$ is the velocity of light. The small perturbations in the centrifugal and Coriolis forces and triaxiality of the bigger primary with the help of parameters are $\psi=1+\varepsilon_{1} ;\left|\varepsilon_{1}\right|<<1, \phi=1+\varepsilon_{2} ;\left|\varepsilon_{2}\right|<<1, \sigma_{i}(i=1,2)$ with $\sigma_{i} \ll<1$, respectively, where $\sigma_{1}=\frac{a^{2}-h^{2}}{5 R^{2}}, \sigma_{2}=\frac{b^{2}-h^{2}}{5 R^{2}}$. (McCuskey [17]) with $\mathrm{a}, \mathrm{b}, \mathrm{h}$ as lengths of its semi-axes and $\mathrm{R}$ is the dimensional distance between the primaries. As Katour et al.[14], we do not include the triaxiality coefficients in the relativistic part since the magnitude of those terms is so small due to $\mathrm{c}^{-2}$, where $\mathrm{c}$ is the speed of light. Consequently, ignoring second and higher powers of $\sigma_{i}, \varepsilon_{i}(i=1,2)$ and also their product, we take equations of motion as:

\section{Locations of triangular points}

The libration points are obtained from equation (1) after putting $\dot{\xi}=\dot{\eta}=\ddot{\xi}=\ddot{\eta}=0$.

These points are the solutions of the equations

$\frac{\partial \mathrm{W}}{\partial \xi}=0=\frac{\partial \mathrm{W}}{\partial \eta}$ with $\dot{\xi}=\dot{\eta}=0$.

The triangular points are the solutions of equations (5) with $\eta \neq 0$. Following as Singh and Bello [16], the coordinates of the triangular points $(\xi, \pm \eta)$ denoted by $\mathrm{L}_{4}$ and $\mathrm{L}_{5}$ respectively are,

$$
\begin{aligned}
& \xi=\frac{1-2 \mu}{2}\left(1+\frac{5}{4 c^{2}}\right)+\left(\frac{1}{8}-\frac{1}{2 \mu}\right) \sigma_{1}+ \\
& \left(\frac{1}{2 \mu}+\frac{3}{8}\right) \sigma_{2}+\left(\frac{-18 \mu^{2}+33 \mu-14}{24(\mu-1) \mathrm{c}^{2}}\right) \varepsilon_{1}, \\
& \eta= \pm\left\{\frac{\sqrt{3}}{2}\left[\begin{array}{c}
1+\frac{1}{12 c^{2}}\left(-5+6 \mu-6 \mu^{2}\right)+ \\
\frac{2}{3}\left\{\left(\frac{1}{2 \mu}-\frac{23}{8}\right) \sigma_{1}+\right. \\
\left(\frac{19}{8}-\frac{1}{2 \mu}\right) \sigma_{2}
\end{array}\right\}\right]+\left(\frac{-2 \sqrt{3}}{9}\right. \\
& +\frac{6 \mu^{2}+6 \mu-5}{54 c^{2}}+ \\
& \left.\left.\frac{\sqrt{3}\left(-10 \mu^{3}+32 \mu^{2}-51 \mu+30\right)}{72(\mu-1) c^{2}}\right)^{\varepsilon_{1}}\right\}
\end{aligned}
$$

\section{Stability of $L_{4}$}

Let $(a, b)$ be the coordinates of the triangular point $\mathrm{L}_{4}$

We set $\xi=a+\alpha, \eta=b+\beta,(\alpha, \beta<<1)$ in the equations (1).

First, we compute the terms of their R.H.S, neglecting second and higher order terms, we obtain 


$$
\left(\frac{\partial \mathrm{W}}{\partial \xi}\right)_{\xi=a+\alpha, \eta=b+\beta}=\mathrm{A} \alpha+\mathrm{B} \beta+\mathrm{C} \dot{\alpha}+\mathrm{D} \dot{\beta}
$$

Where,

$$
\begin{aligned}
& \mathrm{A}=\frac{3}{4}\left\{1+\frac{1}{2 \mathrm{c}^{2}}\left(2-19 \mu+19 \mu^{2}\right)\right\}+ \\
& \frac{3\left(15 \mu^{2}+19 \mu-8\right)}{16 \mu} \sigma_{1}-\frac{3\left(31 \mu^{2}+\mu-8\right)}{16 \mu} \sigma_{2}
\end{aligned}
$$$$
+\left\{\frac{5}{4}+\frac{-31+168 \mu-264 \mu^{2}+126 \mu^{3}}{8(\mu-1) c^{2}}\right\} \varepsilon_{1},
$$$$
\mathrm{B}=\frac{3 \sqrt{3}}{4}(1-2 \mu)\left(1-\frac{2}{3 \mathrm{c}^{2}}\right)-
$$$$
\frac{\sqrt{3}\left(89 \mu^{2}-47 \mu-8\right)}{16 \mu} \sigma_{1}+
$$$$
\frac{\sqrt{3}\left(37 \mu^{2}-9 \mu+8\right)}{16 \mu} \sigma_{2}
$$$$
\left\{\begin{array}{l}
\frac{11 \sqrt{3}(1-2 \mu)}{12}- \\
\frac{\sqrt{3}\left(34-279 \mu+635 \mu^{2}-528 \mu^{3}+134 \mu^{4}\right)}{144(\mu-1) c^{2}}
\end{array}\right\} \varepsilon_{1},
$$$$
C=\frac{\sqrt{3}}{2 c^{2}}(1-2 \mu)+\left\{\frac{5 \sqrt{3}(1-2 \mu)}{18 \mathrm{c}^{2}}\right\} \varepsilon_{1}+\left\{\frac{\sqrt{3}(1-2 \mu)}{2 \mathrm{c}^{2}}\right\} \varepsilon_{2},
$$$$
D=\frac{6-5 \mu+5 \mu^{2}}{2 c^{2}}+\left(\frac{4-15 \mu+15 \mu^{2}}{6 c^{2}}\right) \varepsilon_{1}+\left(\frac{6-5 \mu+5 \mu^{2}}{2 c^{2}}\right) \varepsilon_{2} .
$$

Similarly, we obtain

$$
\left(\frac{\partial W}{\partial \eta}\right)_{\xi=a+\alpha, \eta=b+\beta}=A_{1} \alpha+B_{1} \beta+C_{1} \dot{\alpha}+D_{1} \dot{\beta}
$$

Where,

$$
\begin{aligned}
& \mathrm{A}_{1}=\frac{3 \sqrt{3}}{4}(1-2 \mu)\left(1-\frac{2}{3 \mathrm{c}^{2}}\right)- \\
& \frac{\sqrt{3}\left(89 \mu^{2}-47 \mu+8\right)}{16 \mu} \sigma_{1}+ \\
& \frac{\sqrt{3}\left(37 \mu^{2}-9 \mu+8\right)}{16 \mu} \sigma_{2} \\
& +\left\{\frac{11 \sqrt{3}(1-2 \mu)}{12}-\right. \\
& \left.\frac{\sqrt{3}\left(34-279 \mu+635 \mu^{2}-528 \mu^{3}+132 \mu^{4}\right)}{144(\mu-1) c^{2}}\right\} \varepsilon_{1},
\end{aligned}
$$

$\mathrm{B}_{1}=\frac{9}{4}\left\{1+\frac{7}{6 \mathrm{c}^{2}}\left(-2+3 \mu-3 \mu^{2}\right)\right\}-$

$\frac{3\left(15 \mu^{2}-29 \mu-8\right)}{16 \mu} \sigma_{1}+\frac{3\left(15 \mu^{2}-7 \mu-8\right)}{16 \mu} \sigma_{2}$

$+\left\{\frac{7}{4}+\frac{55-168 \mu+216 \mu^{2}-102 \mu^{3}}{8 c^{2}}\right\} \varepsilon_{1}$,

$\mathrm{C}_{1}=\frac{1}{2 \mathrm{c}^{2}}\left(-4+\mu-\mu^{2}\right)+\left(\frac{-2+\mu-\mu^{2}}{2 \mathrm{c}^{2}}\right) \varepsilon_{1}+\left(\frac{-4+\mu-\mu^{2}}{2 \mathrm{c}^{2}}\right) \varepsilon_{2}$,

$D_{1}=-\frac{\sqrt{3}(1-2 \mu)}{2 c^{2}}+\left\{\frac{-5 \sqrt{3}(1-2 \mu)}{18 c^{2}}\right\} \varepsilon_{1}+\left\{\frac{-\sqrt{3}(1-2 \mu)}{2 c^{2}}\right\} \varepsilon_{2}$.

$\frac{\mathrm{d}}{\mathrm{dt}}\left(\frac{\partial \mathrm{W}}{\partial \dot{\xi}}\right)_{\xi=\mathrm{a}+\alpha, \eta=\mathrm{b}+\beta}=\mathrm{A}_{2} \dot{\alpha}+\mathrm{B}_{2} \dot{\beta}+\mathrm{C}_{2} \ddot{\alpha}+\mathrm{D}_{2} \ddot{\beta}$

Where,

$$
\begin{aligned}
& \mathrm{A}_{2}=\frac{\sqrt{3}}{2 \mathrm{c}^{2}}(1-2 \mu)+\left\{\frac{5 \sqrt{3}(1-2 \mu)}{18 \mathrm{c}^{2}}\right\} \varepsilon_{1}+\left\{\frac{\sqrt{3}(1-2 \mu)}{2 \mathrm{c}^{2}}\right\} \varepsilon_{2}, \\
& \mathrm{~B}_{2}=-\frac{1}{2 \mathrm{c}^{2}}\left(4-\mu+\mu^{2}\right)+\left(\frac{-2+\mu-\mu^{2}}{2 \mathrm{c}^{2}}\right) \varepsilon_{1}+\left(\frac{-4+\mu-\mu^{2}}{2 \mathrm{c}^{2}}\right) \varepsilon_{2}, \\
& \mathrm{C}_{2}=\frac{1}{4 \mathrm{c}^{2}}\left(17-2 \mu+2 \mu^{2}\right)+\left(\frac{1-\mu+\mu^{2}}{2 \mathrm{c}^{2}}\right) \varepsilon_{1}+\left(\frac{10-\mu+\mu^{2}}{2 \mathrm{c}^{2}}\right) \varepsilon_{2}, \\
& \mathrm{D}_{2}=-\frac{\sqrt{3}}{4 \mathrm{c}^{2}}(1-2 \mu)+\left\{\frac{\sqrt{3}(1-2 \mu)}{9 \mathrm{c}^{2}}\right\} \varepsilon_{1}+\left\{\frac{\sqrt{3}(1-2 \mu)}{\left.2 \mathrm{c}^{2}\right\}} \varepsilon_{2} .\right.
\end{aligned}
$$

$\frac{\mathrm{d}}{\mathrm{dt}}\left(\frac{\partial \mathrm{W}}{\partial \dot{\eta}}\right)_{\xi=\mathrm{a}+\alpha, \eta=b+\beta}=\mathrm{A}_{3} \dot{\alpha}+\mathrm{B}_{3} \dot{\beta}+\mathrm{C}_{3} \ddot{\alpha}+\mathrm{D}_{3} \ddot{\beta}$

Where,

$$
\begin{aligned}
& \mathrm{A}_{3}=\frac{1}{2 \mathrm{c}^{2}}\left(6-5 \mu+5 \mu^{2}\right)+\left(\frac{4-15 \mu+15 \mu^{2}}{6 \mathrm{c}^{2}}\right) \varepsilon_{1}+\left(\frac{6-5 \mu+5 \mu^{2}}{2 \mathrm{c}^{2}}\right) \varepsilon_{2}, \\
& \mathrm{~B}_{3}=-\frac{\sqrt{3}}{2 \mathrm{c}^{2}}(1-2 \mu)+\left\{-\frac{5 \sqrt{3}(1-2 \mu)}{18 \mathrm{c}^{2}}\right\} \varepsilon_{1}-\left\{\frac{\sqrt{3}(1-2 \mu)}{2 \mathrm{c}^{2}}\right\} \varepsilon_{2}, \\
& \mathrm{C}_{3}=-\frac{\sqrt{3}}{4 \mathrm{c}^{2}}(1-2 \mu)+\left\{\frac{\sqrt{3}(1-2 \mu)}{9 \mathrm{c}^{2}}\right\} \varepsilon_{1}-\left\{\frac{\sqrt{3}(1-2 \mu)}{\left.4 \mathrm{c}^{2}\right\}} \varepsilon_{2},\right. \\
& \mathrm{D}_{3}=\frac{3\left(5-2 \mu+2 \mu^{2}\right)}{4 \mathrm{c}^{2}}+\left(\frac{7-3 \mu+3 \mu^{2}}{6 \mathrm{c}^{2}}\right) \varepsilon_{1}-\left\{\frac{\sqrt{3}(1-2 \mu)}{4 \mathrm{c}^{2}}\right\} \varepsilon_{2} .
\end{aligned}
$$

The variational equations of motion corresponding to (1), on making use of equation (4), can be expressed as

$$
\mathrm{P}_{1} \ddot{\alpha}+\mathrm{P}_{2} \ddot{\beta}+\mathrm{P}_{3} \dot{\alpha}+\mathrm{P}_{4} \dot{\beta}+\mathrm{P}_{5} \alpha+\mathrm{P}_{6} \beta=0,
$$


Where,

$\mathrm{P}_{1}=1+\mathrm{C}_{2}, \mathrm{P}_{2}=\mathrm{D}_{2}, \mathrm{P}_{3}=\mathrm{A}_{2}-\mathrm{C}, \mathrm{P}_{4}$

$=\left\{\mathrm{B}_{2}-2\left(\begin{array}{c}1+\frac{3}{4}\left(2 \sigma_{1}-\sigma_{2}\right)+\varepsilon_{2}- \\ \frac{3}{2 \mathrm{c}^{2}}\left(1-\frac{1}{3} \mu(1-\mu)\right)\left(1+\varepsilon_{2}\right)\end{array}\right)-\mathrm{D}\right\}$,

$\mathrm{P}_{5}=-\mathrm{A}_{1}, \mathrm{P}_{6}=-\mathrm{B}, \mathrm{q}_{1}=\mathrm{C}_{3}, \mathrm{q}_{2}=1+\mathrm{D}_{3}, \mathrm{q}_{3}$

$=2\left(\begin{array}{l}1+\frac{3}{4}\left(2 \sigma_{1}-\sigma_{2}\right)+\varepsilon_{2} \\ -\frac{3}{2 c^{2}}\left(1-\frac{1}{3} \mu(1-\mu)\right)\left(1+\varepsilon_{2}\right)\end{array}\right)-\mathrm{C}_{1}+\mathrm{A}_{3}, \mathrm{q}_{4}$

$=\mathrm{B}_{3}-\mathrm{D}_{1}, \mathrm{q}_{5}=-\mathrm{A}_{1}, \mathrm{q}_{6}=-\mathrm{B}_{1}$.

Then, the characteristic equation is

$$
\begin{aligned}
& \left(\mathrm{P}_{1} \mathrm{q}_{2}-\mathrm{P}_{2} \mathrm{q}_{1}\right) \lambda^{4}+\left(\mathrm{P}_{1} \mathrm{q}_{6}+\mathrm{P}_{5} \mathrm{q}_{2}+\mathrm{P}_{3} \mathrm{q}_{4}-\mathrm{P}_{6} \mathrm{q}_{1}\right. \\
& \left.-\mathrm{P}_{2} \mathrm{q}_{5}-\mathrm{P}_{4} \mathrm{q}_{3}\right) \lambda^{2}+\mathrm{P}_{5} \mathrm{q}_{6}-\mathrm{P}_{6} \mathrm{q}_{5}=0
\end{aligned}
$$

Substituting the values of $\mathrm{P}_{1}, \mathrm{q}_{\mathrm{i}}, \mathrm{i}=1,2, \ldots, 6$ in (13), the characteristic equation (13) after normalizing becomes

$$
\lambda^{4}+b \lambda^{2}+d=0
$$

Where,

$$
\begin{aligned}
& \mathrm{b}=\left(1-\frac{9}{\mathrm{c}^{2}}\right)+3 \sigma_{1}+\frac{3}{2}(2 \mu-3) \sigma_{2}+ \\
& \left\{-3+\frac{68-25 \mu+25 \mu^{2}}{4 \mathrm{c}^{2}}\right\} \varepsilon_{1} \\
& +\left\{8+\frac{-147+30 \mu-30 \mu^{2}}{2 \mathrm{c}^{2}}\right\} \varepsilon_{2}, \\
& \mathrm{~d}=\frac{27 \mu(1-\mu)}{4}+\frac{9 \mu\left(-65+77 \mu-24 \mu^{2}+12 \mu^{3}\right)}{8 \mathrm{c}^{2}} \\
& +\frac{9\left(-10+99 \mu-89 \mu^{2}\right)}{16} \sigma_{1}+\frac{9\left(10-47 \mu+37 \mu^{2}\right)}{16} \sigma_{2} \\
& +\left\{\frac{33 \mu(1-\mu)}{2}-\frac{3 \mu\left(1867-2082 \mu+540 \mu^{2}-336 \mu^{3}\right.}{32 c^{2}}\right\} \varepsilon_{1} \\
& +\left\{\frac{-243 \mu+234 \mu^{2}-162 \mu^{3}+81 \mu^{4}}{4 \mathrm{c}^{2}}\right\} \varepsilon_{2} . \\
& +\left\{\begin{array}{l}
16 \\
4
\end{array}\right.
\end{aligned}
$$

For $\frac{1}{c^{2}} \rightarrow 0$ and in the absence of small perturbations (in the centrifugal and Coriolis forces) and triaxiality (i.e. $\sigma_{1}=\sigma_{2}=\varepsilon_{1}=\varepsilon_{2}=0$ ), (9) reduces to its well-known classical restricted problem form (see e.g. Szebehely [1]): $\lambda^{4}+\lambda^{2}+\frac{27}{4} \mu(1-\mu)=0$

The discriminant of (9) is

$$
\begin{aligned}
& \Delta=\left(\frac{-54}{c^{2}}-\frac{126}{c^{2}} \varepsilon_{1}-\frac{81}{c^{2}} \varepsilon_{2}\right) \mu^{4} \\
& +\left(\frac{108}{c^{2}}+\frac{405}{2 c^{2}} \varepsilon_{1}-\frac{162}{c^{2}} \varepsilon_{2}\right) \mu^{3} \\
& +\left(27+\frac{801}{4} \sigma_{1}-\frac{333}{4} \sigma_{2}+66 \varepsilon_{1}-\frac{3073}{4 c^{2}} \varepsilon_{1}\right. \\
& \left.-\frac{354}{c^{2}} \varepsilon_{2}-\frac{693}{2 c^{2}}\right) \mu^{2}
\end{aligned}
$$$$
+\left(\begin{array}{l}
-27-\frac{891}{4} \sigma_{1}+\frac{447}{4} \sigma_{2}-66 \varepsilon_{1} \\
+\frac{5501}{8 c^{2}} \varepsilon_{1}+\frac{273}{c^{2}} \varepsilon_{2}+\frac{585}{2 c^{2}}
\end{array}\right) \mu+1+\frac{57}{2} \sigma_{1}-\frac{63}{2} \sigma_{2}
$$$$
-6 \varepsilon_{1}+\frac{88}{\mathrm{c}^{2}} \varepsilon_{1}+16 \varepsilon_{2}-\frac{18}{\mathrm{c}^{2}}-\frac{291}{\mathrm{c}^{2}} \varepsilon_{2}
$$

Its roots are

$$
\lambda^{2}=\frac{-\mathrm{b} \pm \sqrt{\Delta}}{2}
$$

Where,

$$
\begin{aligned}
& \mathrm{b}=\left(1-\frac{9}{\mathrm{c}^{2}}\right)+3 \sigma_{1}+\frac{3}{2}(2 \mu-3) \sigma_{2}+ \\
& \left\{-3+\frac{68-25 \mu+25 \mu^{2}}{4 \mathrm{c}^{2}}\right\} \varepsilon_{1}+\left\{8+\frac{-147+30 \mu-30 \mu^{2}}{2 \mathrm{c}^{2}}\right\} \varepsilon_{2},
\end{aligned}
$$

From (10), we have

$$
\begin{aligned}
& \frac{\mathrm{d} \Delta}{\mathrm{d} \mu}=4\left(\frac{-54}{\mathrm{c}^{2}}-\frac{126}{\mathrm{c}^{2}} \varepsilon_{1}-\frac{81}{\mathrm{c}^{2}} \varepsilon_{2}\right) \mu^{3} \\
& +3\left(\frac{108}{\mathrm{c}^{2}}+\frac{405}{2 \mathrm{c}^{2}} \varepsilon_{1}+\frac{162}{\mathrm{c}^{2}} \varepsilon_{2}\right) \mu^{2} \\
& +2\left(27+\frac{801}{4} \sigma_{1}-\frac{333}{4} \sigma_{2}+66 \varepsilon_{1}\right. \\
& \left.-\frac{3073}{4 \mathrm{c}^{2}} \varepsilon_{1}-\frac{354}{\mathrm{c}^{2}} \varepsilon_{2}-\frac{693}{2 \mathrm{c}^{2}}\right) \mu \\
& +\left(\begin{array}{l}
-27-\frac{891}{4} \sigma_{1}+\frac{447}{4} \sigma_{2}- \\
\left.66 \varepsilon_{1}+\frac{5501}{8 \mathrm{c}^{2}} \varepsilon_{1}+\frac{273}{\mathrm{c}^{2}} \varepsilon_{2}+\frac{585}{2 \mathrm{c}^{2}}\right)<0
\end{array}\right.
\end{aligned}
$$

$$
\text { for } \mu \in\left(0, \frac{1}{2}\right)
$$

From (12), it can be easily seen that $\Delta$ is monotone decreasing in $\left(0, \frac{1}{2}\right)$ 
But

$$
\begin{aligned}
& (\Delta)_{\mu=0}=1+\frac{57}{2} \sigma_{1}-\frac{63}{2} \sigma_{2}-6 \varepsilon_{1} \\
& +\frac{88}{c^{2}} \varepsilon_{1}+16 \varepsilon_{2}-\frac{291}{c^{2}} \varepsilon_{2}-\frac{18}{c^{2}}>0 \\
& (\Delta)_{\mu=\frac{1}{2}}=-\frac{23}{4}+\frac{207}{4 c^{2}}-\frac{525}{16} \sigma_{1}+\frac{57}{16} \sigma_{2} \\
& +\frac{4115}{16 c^{2}} \varepsilon_{1}+16 \varepsilon_{2}-\frac{3645}{16 c^{2}} \varepsilon_{2}<0
\end{aligned}
$$

Since $(\Delta)_{\mu=0}$ and $(\Delta)_{\mu=\frac{1}{2}}$ are of opposite signs, and $\Delta$ is monotone decreasing and continuous, there is one value of $\mu$, e.g. $\mu_{c}$ in the interval $\left(0, \frac{1}{2}\right)$ for which $\Delta$ vanishes.

Solving the equation $\Delta=0$, using (10), we obtain the critical value of the mass parameter as

$$
\begin{aligned}
& \mu_{\mathrm{c}}=\frac{1}{2}-\frac{1}{18} \sqrt{69}-\frac{17 \sqrt{69}}{486 \mathrm{c}^{2}}+\frac{1}{2}\left(\frac{5}{6}+\frac{59}{9 \sqrt{69}}\right) \sigma_{1} \\
& -\frac{1}{2}\left(\frac{19}{18}+\frac{85}{9 \sqrt{69}}\right) \sigma_{2}+\frac{4\left(36 \varepsilon_{2}-19 \varepsilon_{1}\right)}{27 \sqrt{69}} \\
& +\left(\frac{34155+175301 \sqrt{69}}{804816 \mathrm{c}^{2}}\right) \varepsilon_{1}-\left(\frac{47 \sqrt{69}}{81 \mathrm{c}^{2}}\right) \varepsilon_{2} \\
& \mu_{\mathrm{c}}=\mu_{0}-\frac{17 \sqrt{69}}{486 \mathrm{c}^{2}}+\frac{1}{2}\left(\frac{5}{6}+\frac{59}{9 \sqrt{69}}\right) \sigma_{1} \\
& -\frac{1}{2}\left(\frac{19}{18}+\frac{85}{9 \sqrt{69}}\right) \sigma_{2}+\frac{4\left(36 \varepsilon_{2}-19 \varepsilon_{1}\right)}{27 \sqrt{69}} \\
& \left(\frac{34155+175301 \sqrt{69}}{804816 \mathrm{c}^{2}}\right) \varepsilon_{1}-\left(\frac{47 \sqrt{69}}{81 \mathrm{c}^{2}}\right) \varepsilon_{2}
\end{aligned}
$$

Where $\mu_{0}=0.03852 \ldots$ is the Routh's value.

We consider the following three regions of the values of $\mu$ separately.

i) When $0 \leq \mu<\mu_{c} \Delta>0$, the values of $\lambda^{2}$ given by (11) are negative and therefore all the four characteristic roots are distinct pure imaginary numbers. Hence, the triangular points are stable.

ii) When $\mu_{\mathrm{c}}<\mu \leq \frac{1}{2}, \Delta<0$, the real parts of the characteristic roots are positive. Therefore, the triangular points are unstable.

iii) When $\mu=\mu_{\mathrm{c}}, \Delta=0$, the values of $\lambda^{2}$ given by (11) are the same. Hence the solution contains secular terms. This induces instability of the triangular points.

Hence, the stability region is

$$
\begin{aligned}
& 0 \leq \mu<\mu_{0}-\frac{17 \sqrt{69}}{486 \mathrm{c}^{2}}+\frac{1}{2}\left(\frac{5}{6}+\frac{59}{9 \sqrt{69}}\right) \sigma_{1} \\
& -\frac{1}{2}\left(\frac{19}{18}+\frac{85}{9 \sqrt{69}}\right) \sigma_{2}+\frac{4\left(36 \varepsilon_{2}-19 \varepsilon_{1}\right)}{27 \sqrt{69}} \\
& +\left(\frac{34155+175301 \sqrt{69}}{804816 \mathrm{c}^{2}}\right) \varepsilon_{1}-\left(\frac{47 \sqrt{69}}{81 \mathrm{c}^{2}}\right) \varepsilon_{2}
\end{aligned}
$$

\section{Discussion}

We now discuss the triangular libration points in the perturbed relativistic R3BP under the assumption that the bigger primary is a triaxial rigid body. In analogy to corresponding problem without perturbations and triaxiality, the positions of analogous triangular libration points (6) are obtained. It is important to note that these triangular libration points (6) cease to be classical ones i.e. they no longer form equilateral triangles with the primaries as they do in the classical case. Rather they form scalene triangles with the primaries. It is seen from (6), that the positions of triangular points are affected by the relativistic effect, triaxiality and the perturbation in the centrifugal force, but not affected by that of the Coriolis force.

Equation (14) gives the critical value of the mass parameter $\mu_{\mathrm{c}}$ of the system which depends upon triaxiality, relativistic factor and small perturbations $\varepsilon_{1}, \varepsilon_{2}$ in the centrifugal and Coriolis forces, respectively. This critical value is used to determine the size of the region of stability of the triangular points and also helps in analyzing the behavior of the parameters involved therein. It is obvious from (15) that the relativistic and triaxiality effects reduce the size of the stability region separately where as the Coriolis effect expands it if $\varepsilon_{2}>0$ and shrinks it if $\varepsilon_{2}<0$. similarly the separate effect of centrifugal force expands it if $\varepsilon_{1}<0$ and shrinks it if $\varepsilon_{1}>0$.

Even on considering the coupling terms $\frac{\varepsilon_{\mathrm{i}}}{\mathrm{c}^{2}}(\mathrm{i}=1,2)$ which are very small quantities, from mathematical point of view, it can be seen that from (15) that the joint effect of the relativistic term and centrifugal force i.e. the term containing the coupling term $\frac{\varepsilon_{1}}{\mathrm{c}^{2}}$ expands the size of the stability region if $\varepsilon_{1}>0$ and shrinks it if $\varepsilon_{1}<0$; whereas the joint effect of the relativistic and Coriolis force i.e. the term containing the coupling term $\frac{\varepsilon_{2}}{\mathrm{c}^{2}}$ shrinks it if $\varepsilon_{2}>0$ and expands it if $\varepsilon_{2}<0$.

From the overall analysis, it is clear that the Coriolis and centrifugal forces maintained their stabilizing and destabilizing characteristic behavior respectively.

However, it can be seen that from equation (15) that the net effect is that the size of the range of stability increases or decreases according as equation (14) without $\mu_{0}$ is greater or less than zero.

In the absence of triaxiality (i.e. $\sigma_{1}=\sigma_{2}=0$ ) and a small perturbation in the Coriolis force (i.e. $\varepsilon_{2}=0$ ), the results of this study are in agreement with those of Singh and Bello [15].When the bigger primary is spherical (i.e. $\sigma_{1}=\sigma_{2}=0$ ) and the coupling terms $\frac{\varepsilon_{i}}{c^{2}}(i=1,2)$ are neglected, the results of the present study are in accordance with those of Singh and Bello [16].

In the absence of relativistic terms, our result coincides with those of Singh [9] when the primaries are non-luminous and the bigger triaxial only. 


\section{Conclusion}

Under the assumption that the bigger primary is a triaxial rigid body and small perturbations $\varepsilon_{1}, \varepsilon_{2}$ are given to the centrifugal and Coriolis forces, the stability of the triangular equilibrium points in the relativistic R3BP has been examined. It is found that their positions are affected by the relativistic factor, a small change in the centrifugal force and triaxiality factors of the bigger primary. It is also observable from equation (19) that all the parameters involved in this study except the Coriolis force have destabilizing tendencies resulting in a decrease in the size of the region of stability.

It is also noticed that the expressions for $\mathrm{A}, \mathrm{D}, \mathrm{A}_{2}, \mathrm{C}_{2}$ in Bhatnagar and Hallan [12] differ from the present unperturbed study. Consequently, the expressions $\mathrm{P}_{1}, \mathrm{P}_{3}, \mathrm{P}_{4}, \mathrm{P}_{5}$ and the characteristic equation are also different. This led them (Bhatnagar and Hallan [12]) to infer that the triangular points are unstable, contrary to Douskos and Perdios and our results.

A practical application of this model could be the study of the motion of a dust grain particle near Pluto and its moon Charon.

\section{References}

[1] Szebehely V (1967), Theory of orbits. The restricted problem of three- bodies. Academic Press. New York.

[2] Wintner A (1941), The Analytical foundations of celestial mechanics, Princeton University press, Princeton

[3] Contopolous G (2002), Order and Chaos in Dynamical Astronomy Spring, Berlin. http://dx.doi.org/10.1007/978-3-662-04917-4.

[4] Szebehely V (1967), Stability of the points of equilibrium in the restricted problem, Astronomical Journal, 27, 7-9. http://dx.doi.org/10.1086/110195.

[5] Bhatnagar KB\& Hallan PP (1978), Effect of perturbations in the Coriolis and centrifugal forces on the stability of libration points in restricted problem,Celestial Mechanics, 18,105-112. http://dx.doi.org/10.1007/BF01228710.

[6] AbdulRaheem A \& Singh J (2006), Combined effects of perturbations, radiation and oblateness on the stability of equilibrium points in the restricted three-body problem,Atronomical Journal,131, 1880-1885. http://dx.doi.org/10.1086/499300.

[7] Singh J \&Begha JM (2011), Stability of equilibrium points in the generalized perturbed restricted three-body problem, Astrophysics \& Space Science, 331, 511-519. http://dx.doi.org/10.1007/s10509010-0464-1.

[8] Abouelmagd E I,Asiri HM \& Sharaf MA (2013), The effects of oblateness in the perturbed restricted three-body problem, Meccanica,48, 2479-2490. http://dx.doi.org/10.1007/s11012-013-9762$\underline{3}$.

[9] Singh J (2013), the equilibrium points in the perturbed R3BP with triaxial and luminous primaries Astrophys \& Space Science, 346, 41-50. http://dx.doi.org/10.1007/s10509-013-1420-7.

[10] Brumberg VA (1972), Relativistic Celestial Mechanics. Moscow, Nauka.

[11] Brumberg VA (1991), Essential Relativistic Celestial Mechanics. Adam Hilger Ltd, New York.

[12] Bhatnagar KB \&Hallan PP (1998), Existence and stability of L ${ }_{4,5}$ in the relativistic restricted three-body problem. Celestial Mechanics \&Dynamical Astronomy, 69, 271-281 http://dx.doi.org/10.1023/A:1008271021060.

[13] Douskos CN \&Perdios EA (2002), "On the stability of equilibrium points in the relativistic restricted three-body problem", Celestial Mechanics \&Dynamical Astronomy, 82, 317-321. http://dx.doi.org/10.1023/A:1015296327786.

[14] Katour DA, Abd El-Salam FA \& Shaker MO (2014), Relativistic restricted three-body problem with oblateness and photogravitational corrections to triangular equilibrium points, Astrophysics \& Space Science, 351(1), 143-149. http://dx.doi.org/10.1007/s10509-014-1826-x.

[15] Singh J \& Bello N (2014), Motion around $\mathrm{L}_{4}$ in the perturbed relativistic R3BP, Astrophysics \& Space Science, 351(2), 491-497. http://dx.doi.org/10.1007/s10509-014-1870-6.

[16] Singh J \& Bello N (2014), Effect of perturbations in the Coriolis and centrifugal forces on the stability of $\mathrm{L}_{4}$ in the relativistic R3BP
Journal of Astrophysics \&Astronomy, 35 (4) 701-713. http://dx.doi.org/10.1007/s12036-014-9307-8.

[17] McCuskey SW (1963), Introduction to celestial mechanics, Addison, Wesley. 